\title{
The Effects of Pyridine and Pyrazine Carboxylic Acids Derivatives on the Growth of Lemna paucicostata 151
}

\author{
Hiroshi Taguchi, ${ }^{\dagger}$ Atsushi Kashimoto, Hiroshi Nishitani, \\ Yoshihide SHImabaYashi and Kazuo IWAi* \\ Department of Agricultural Chemistry, Faculty of Agriculture, Mie University, Tsu, Mie 514, Japan \\ * Department of Food Science and Technology, Faculty of Agriculture, Kyoto University, \\ Sakyo-ku, Kyoto 606, Japan \\ Received July 8, 1987
}

\begin{abstract}
The effects of pyridine and pyrazine carboxylic acids derivatives on growth, floral induction, and the contents of niacin and NAD in the short-day plant Lemna paucicostata 151 were investigated. Among the compounds which promoted plant growth, cinchomeronic acid and trigonelline were the most effective. Picolinic acid was the strongest inhibitor of plant growth among tested. These effects may be concerned with the biosynthesis and metabolism of NAD. The results obtained in this study suggested that the substituents on the third position of the pyridine ring require large volume and high electronegativity to promote plant growth. Floral induction was observed with nicotinamide, isonicotinamide, pyrazinamide, etc.
\end{abstract}

A number of species in the family Lemnaceae provide much excellent material for studying growth and flowering, because of their minute size, rapid growth and easy manipulation under aseptic conditions. Various hormones, ${ }^{1 \sim 3)}$ sulfhydryl inhibitors, ${ }^{4 \sim 9)}$ adenine derivatives ${ }^{10,11)}$ and amino $\operatorname{acids}^{12,13 \text { ) }}$ have been reported to be involved in floral induction in Lemnaceae. Furthermore, benzoic acid derivatives have also been reported to induce flowering in Lemna paucicostata 151. ${ }^{14 \sim 17)}$ Watanabe et al. ${ }^{16)}$ reported that of pyridine derivatives, picolinic acid, nicotinic acid and isonicotinic acid are involved in floral induction in L. paucicostata 151 cultured with $1 / 10$ strength $\mathrm{M}$ medium containing $1 \%$ sucrose under continuous illumination. Also, nicotinamide has been shown as a plant growth regulator to some crops. ${ }^{18 \text { ) }}$

On the basis of these aspects and the structural similarity between the pyridine ring and the pyrazine ring, we examined the effects of these compounds on growth, floral induction and the content of niacin and NAD in $L$. paucicostata 151.

\section{MATERIALS AND METHODS}

Lemna assay. L. paucicostata 151 was used in all experiments under aseptic conditions. Stock cultures were grown in $100 \mathrm{ml}$ Erlenmeyer flasks containing $40 \mathrm{ml}$ of $1 / 2$ strength Hutner's medium ${ }^{19\}}$ with $1 \%$ sucrose under continuous illumination with daylight-type fluorescent lamps of about $4000 \mathrm{lux}$ at $25 \pm 1^{\circ} \mathrm{C}$ in an NK System Biotron, type LH-100-RD, Nippon Medical \& Chemical Instruments Co., Ltd., Tokyo. This medium was sterilized by autoclaving at $1.2 \mathrm{~kg} / \mathrm{cm}^{2}$ for $10 \mathrm{~min}$ after adjusting to $\mathrm{pH}$ 4.7. All experimental cultures with test compounds were grown in $1 / 10$ strength $\mathrm{M}$ medium ${ }^{20}$ containing $1 \%$ sucrose, which was sterilized under the same conditions after adjusting to $\mathrm{pH}$ 4.6. When cupric ion was added to the experimental culture medium, the final concentration was always $8 \mu \mathrm{g} / 1$ as $\mathrm{CuSO}_{4} \cdot 5 \mathrm{H}_{2} \mathrm{O}$.

One three-frond colony of L. paucicostata 151 , from 7 to 10 days old on the stock culture, was transplanted to a $100 \mathrm{ml}$ Erlenmeyer flask containing the experimental medium in which each test compound was contained at varying concentrations. All experimental cultures were grown for 7 days under the conditions mentioned above. Then the growth rate relative to growth in the control experiment without test compound and flowering percentage were calculated. Each experiment was duplicated

\footnotetext{
$\dagger$ To whom correspondence should be addressed.
} 
and repeated at least twice. Average values obtained from such experiments are shown below.

Extraction of niacin and NAD. Extraction and fractionation of the compounds from $L$. paucicostata 151 at floral induction were done by the methods of Imai and Suzuki. ${ }^{21)}$

Niacin and NAD assay. Niacin and NAD were measured by a microbiological assay with Lactobacillus plantarum ATCC $8014 .{ }^{22)}$

Chemicals. All chemicals used in these experiments were the highest grade among those usually obtainable and purchased through Tokyo Kasei Kogyo Co., Ltd., Tokyo and Nakarai Chemicals Ltd., Kyoto.

\section{RESULTS AND DISCUSSION}

Effects on growth

Table I shows the effects of pyridine and pyrazine derivatives on growth at various concentrations. These effects were affected by cupric ion which is a well-known inhibitor of SH-enżymes, e.g., nitrate reductase (EC 1.6.6.3). ${ }^{23)}$ When the cupric ion was added to the control medium, the rate of plant growth was slightly lowered to about $94 \%$. Among the compounds which promoted growth, cinchomeronic acid (pyridine-3,4-dicarboxylic acid) and trigonelline ( $N$-methylnicotinic acid) were

Table I. Effects of Pyridine and Pyrazine Carboxylic Acids Derivatives on the Growth and Flowering of Lemna paucicostata 151 wITH OR WITHOUT CuPRIC ION

Values shown are growth rate relative to growth in the control experiment without test compound. " $F$ " beside the value means that floral induction was observed.

\begin{tabular}{|c|c|c|c|c|c|c|}
\hline \multirow{3}{*}{ Compound } & \multicolumn{3}{|c|}{ With cupric ion } & \multicolumn{3}{|c|}{ Without cupric ion } \\
\hline & \multicolumn{3}{|c|}{ Concentration (M) } & \multicolumn{3}{|c|}{ Concentration (M) } \\
\hline & $10^{-5}$ & $10^{-4}$ & $10^{-3}$ & $10^{-5}$ & $10^{-4}$ & $10^{-3}$ \\
\hline \multicolumn{7}{|l|}{ Pyridine derivatives } \\
\hline (1) $2-\mathrm{COOH}$ & 0.64 & 0.12 & 0.00 & 0.61 & 0.13 & 0.00 \\
\hline (2) $3-\mathrm{COOH}$ & 1.22 & $0.58 \mathrm{~F}$ & 0.08 & 0.84 & $0.40 \mathrm{~F}$ & 0.08 \\
\hline (3) $4-\mathrm{COOH}$ & 0.79 & $0.52 \mathrm{~F}$ & 0.12 & 0.89 & $0.33 \mathrm{~F}$ & 0.08 \\
\hline (4) $2-\mathrm{CONH}_{2}$ & 0.82 & 0.84 & 0.52 & 1.08 & 0.86 & 1.04 \\
\hline (5) $3-\mathrm{CONH}_{2}$ & 0.76 & $0.42 \mathrm{~F}$ & $0.41 \mathrm{~F}$ & $0.81 \mathrm{~F}$ & $0.45 \mathrm{~F}$ & $0.30 \mathrm{~F}$ \\
\hline (6) $4-\mathrm{CONH}_{2}$ & 1.11 & 0.67 & 1.17 & 1.19 & $0.64 \mathrm{~F}$ & 0.98 \\
\hline (7) $2,3-(\mathrm{COOH})_{2}$ & 0.96 & 1.19 & 0.83 & 0.94 & 0.96 & 0.66 \\
\hline (8) $2,4-(\mathrm{COOH})_{2}$ & 0.84 & 0.83 & 0.00 & 0.61 & 0.80 & 0.00 \\
\hline (9) $2,5-(\mathrm{COOH})_{2}$ & 0.83 & 1.12 & 1.04 & 0.85 & 1.04 & 0.78 \\
\hline (10) $2,6-(\mathrm{COOH})_{2}$ & 0.90 & 1.29 & 0.00 & 1.37 & 1.34 & 0.00 \\
\hline (11) $3,4-(\mathrm{COOH})_{2}$ & 1.20 & 1.61 & 1.34 & 1.31 & 1.67 & 1.93 \\
\hline (12) $3,5-(\mathrm{COOH})_{2}$ & 1.15 & 1.04 & 0.88 & 1.30 & 1.28 & 1.11 \\
\hline (13) $1-\mathrm{CH}_{3}, 3-\mathrm{COOH}$ & 1.75 & 1.66 & 1.42 & 1.17 & 1.43 & 1.26 \\
\hline (14) $3-\mathrm{CONHCH}_{3}$ & 1.34 & 1.33 & 0.67 & 1.01 & 0.89 & 0.80 \\
\hline (15) 3-CON $\left(\mathrm{CH}_{3}\right)_{2}$ & 1.65 & 1.15 & 1.17 & 1.52 & 1.05 & 1.47 \\
\hline (16) 3-CONH ${ }_{2}, 6-\mathrm{NH}_{2}$ & 1.38 & 1.14 & 0.81 & 1.23 & 1.04 & 0.68 \\
\hline (17) Pyridoxine & 1.21 & 1.19 & 1.17 & 0.87 & 0.89 & 0.83 \\
\hline (18) Pyridoxal & 1.13 & 1.13 & 0.00 & 0.80 & 1.18 & 0.00 \\
\hline (19) Pyridoxamine & 1.33 & 0.74 & 0.85 & 0.95 & 1.12 & 0.81 \\
\hline \multicolumn{7}{|l|}{ Pyrazine derivatives } \\
\hline (20) Pyrazine & 1.39 & 0.83 & 1.11 & 0.98 & 0.78 & 0.65 \\
\hline (21) $2-\mathrm{COOH}$ & 0.79 & $0.36 \mathrm{~F}$ & $0.21 F$ & 0.93 & $0.38 \mathrm{~F}$ & $0.19 \mathrm{~F}$ \\
\hline (22) $2-\mathrm{CONH}_{2}$ & $0.37 \mathrm{~F}$ & $0.28 \mathrm{~F}$ & 0.07 & $0.93 \mathrm{~F}$ & $0.33 \mathrm{~F}$ & 0.16 \\
\hline (23) $2,3-(\mathrm{COOH})_{2}$ & 1.05 & 0.95 & $0.49 \mathrm{~F}$ & 1.14 & 0.73 & $0.4 \mathrm{IF}$ \\
\hline
\end{tabular}

Trivial names are: (1) picolinic acid; (2) nicotinic acid; (3) isonicotinic acid; (4) picolinamide; (5) nicotinamide; (6) isonicotinamide; (7) quinolinic acid; (8) Iutidinic acid; (10) dipicolinic acid; (11) cinchomeronic acid; (13) trigonelline. 


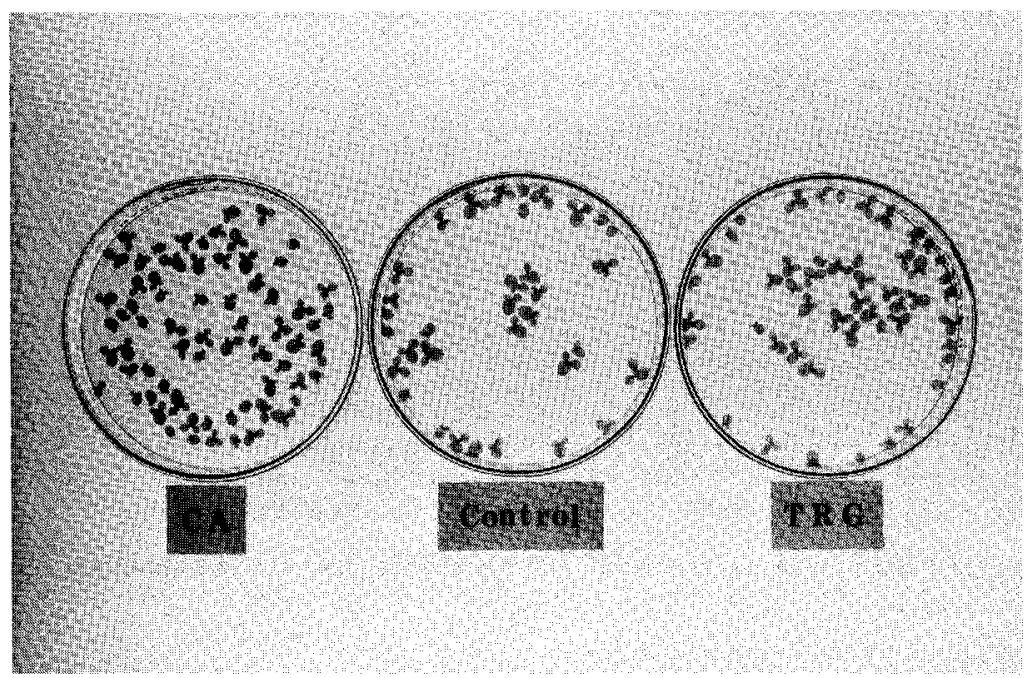

FIG. 1. Growth Promotion of Lemna paucicostata 151 by Cinchomeronic Acid and Trigonelline without Cupric lon.

Left: in cinchomeronic acid containing medium at $10^{-3} \mathrm{M}$. Center: control. Right: in trigonelline containing medium at $10^{-4} \mathrm{M}$.

much more effective than the others. A photograph of a representative result is shown in Fig. 1. These effects may be concerned with the biosynthesis and metabolism of NAD, because both compounds can structurally be derived from nicotinic acid. In this connection, trigonelline synthesizing enzyme (nicotinate methyltransferase) activity was found widely in plants and the activity was high. ${ }^{24)}$ Also trigonelline may have a hormone-like action on plant tissues. ${ }^{25)}$ When the plant was grown in cinchomeronic-acid-containing medium, the leaf was much thicker and the green color was much darker than those grown in other medium (compare the darkness of leaves in Fig. 1). These results suggested that cinchomeronic acid greatly affected the physiology of $L$. paucicostata 151 . As far as we know, the plant growth promoting effect of cinchomeronic acid was found for the first time in this experiment. This effect was also found in pea seedlings, Escherichia coli, etc. (our unpublished data, to be published) but how it works is not known.

Dipicolinic acid (pyridine-2,6-dicarboxylic acid) is a chelating agent and an inhibitor for

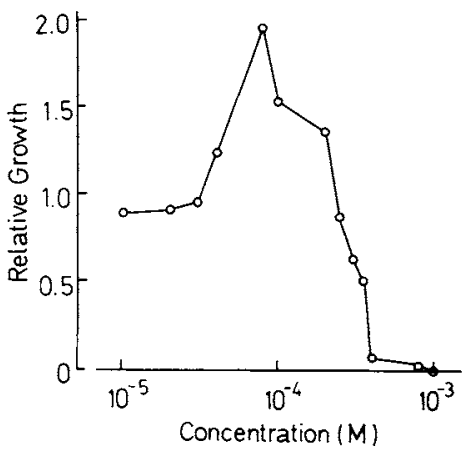

FIG. 2. Effects of Dipicolinic Acid on the Growth of Lemna paucicostata 151 with Cupric Ion.

metal-requiring enzymes, e.g., quinolinate phosphoribosyltransferase (EC 2.4.2.19) ${ }^{26}$ ) which is a key enzyme in the de novo biosynthetic pathway of NAD. This compound made multiplication rate of the plant faster and the leaf area larger below $10^{-4} \mathrm{M}$. These effects were independent of cupric ion, but on higher levels of application of dipicolinic acid in the medium, the effect disappeared and the plants died. As shown in Fig. 2, this growthpromoting effect was observed only within a narrow range of the concentration. Among the 

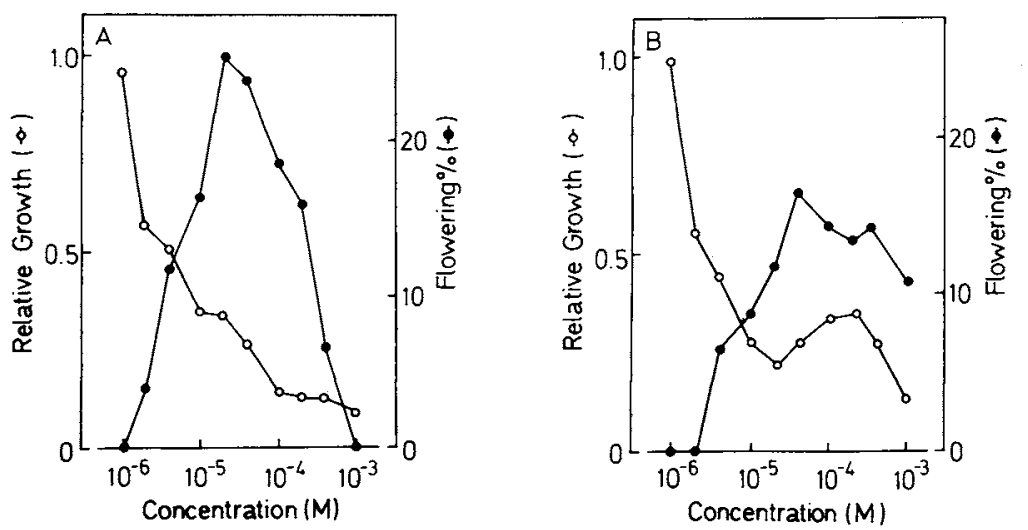

FIG. 3. Effect of Pyrazinamide on the Growth and Floral Induction of Lemna paucicostata 151 with or without Cupric Ion.

A: with cupric ion. B: without cupric ion.

compounds which inhibited growth, picolinic acid (pyridine-2-carboxylic acid) was the strongest. These results suggested that the substituents on the third-position of pyridine ring require large volume and high electronegativity to promote plant growth. Detailed study of quantitative structure-activity relationships might find the most favorable structure of the compound for plant growth promotion. Also, the nicotinamide derivatives, e.g., dimethylnicotinamide (compound No. 15 in Table I), tended to promote the plant growth. It seems that they inhibited the biosynthetic and metabolic pathways of NAD.

Incidentally Taguchi et al. found that picolinic acid and isonicotinic acid are competitive inhibitors, and nicotinamide is a noncompetitive inhibitor of a partially purified nicotinate methyltransferase (EC 2.1.1.7) from a marine clam. ${ }^{27)}$ Nicotinic acid metabolism may be regulated by these compounds in marine clams. It is probable that a similar regulatory mechanism is present in plants. To clarify this problem, extensive enzymological study is required.

\section{Floral induction with pyrazinamide}

Pyrazinamide, which is a well known antituberculosis drug, inhibited the NAD metabolic pathway in rats. ${ }^{28)}$ On the other hand, no report has been published on pyrazinamide as
Table II. NiaCin and NAD Contents in Lemna paucicostata 151 at Floral Induction Grown WITHOUT CUPRIC ION

\begin{tabular}{lrrc}
\hline $\begin{array}{l}\text { Compound added } \\
\text { to the medium }\end{array}$ & Niacin* & NAD $^{*}$ & $\begin{array}{c}\text { Floral } \\
\text { induction }\end{array}$ \\
\hline Control & 2.7 & 31.7 & - \\
Nicotinamide $\left(10^{-4} \mathrm{M}\right)$ & 73.9 & 136.5 & + \\
Pyradinamide $\left(10^{-4} \mathrm{M}\right)$ & 7.7 & 17.7 & + \\
Pyradinamide $\left(10^{-5} \mathrm{M}\right)$ & 5.7 & 51.2 & + \\
\hline
\end{tabular}

* Nicotinic acid equivalent $(\mu \mathrm{g} / \mathrm{g}$ fresh weight).

a plant growth regulator. Figure 3 shows the relationship between floral induction and the concentration of pyrazinamide added to $1 / 10$ strength $\mathrm{M}$ medium containing $1 \%$ sucrose with or without cupric ion. Floral induction depended on the existence of cupric ion and the amount of pyrazinamide. In the cupricion-containing medium, floral induction was observed up to higher concentrations of pyrazinamide. The result suggested that pyrazinamide or its derivatives may affect the biosynthesis and biodegradation of NAD.

\section{Relationship between the flowering and the con- tent of niacin or $N A D$}

As shown in Table II, niacin content was lower than the NAD content. Niacin may be easily incorporated into NAD in the plant cell. The content of NAD in flowering plants grown 
in nicotinamide-containing medium at $10^{-4} \mathrm{M}$ was 4.3 times higher than that of control. In the addition of pyrazinamide at $10^{-4} \mathrm{M}$, the NAD content was lowered to about one half that of the control. At $10^{-5} \mathrm{M}$ pyrazinamide, the content was 1.6 times higher than that of the control. These results suggested that the biosynthesis and metabolism of NAD in the plant were disturbed when niacin-related compounds were added to the medium, but no direct relationship was observed between floral induction and niacin or NAD content. Localization of NAD, or the concentration of NAD metabolites (e.g., poly-ADP-ribose), or the ratio of specified compound to NAD, or the concentration of niacin metabolite (e.g., trigonelline), etc. may directly control growth and flowering. The relationship between plant growth and flowering is also uncertain. Further investigation is under progress now to solve these problems.

Acknowledgments. We wish to express our hearty gratitude to Professor Dr. Atsushi Takimoto, Faculty of Agriculture, Kyoto University, for supplying Lemna paucicostata 151 . We also wish to express many thanks to Professor Dr. Nobutaka Takahashi, Faculty of Agriculture, Tokyo University, for kind advice and suggestions.

\section{REFERENCES}

1) S. Gupta and S. C. Maheshwari, Plant \& Cell Physiol., 11, 97 (1970).

2) J. M. Ives and H. B. Posner, Plant Physiol, 70, 311 (1982).

3) S. Fujioka, I. Yamaguchi, N. Murofushi, N. Takahashi, S. Kaihara and A. Takimoto, Plant \& Cell Physiol., 24, 241 (1983).

4) W. S. Hillman, Am. J. Bot., 49, 892 (1962).

5) A. Takimoto and O. Tanaka, Plant \& Cell Physiol., 14, 1133 (1973).
6) A. Takimoto and O. Tanaka, Plant \& Cell Physiol., 17, 299 (1976).

7) O. Tanaka and A. Takimoto, Plant \& Cell Physiol., 19, 701 (1978).

8) O. Tanaka, A. Takimoto and C. F. Cleland, Plant \& Cell Physiol., 20, 267 (1979)

9) O. Tanaka, C. F. Cleland and Y. Ben-Tal, Plant \& Cell Physiol., 24, 705 (1983).

10) Y. Oota, Plant \& Cell Physiol., 13, 195 (1972).

11) H. B. Posner, Plant \& Cell Physiol, 14, 1199 (1973).

12) H. B. Posner, Plant Physiol., 48, 361 (1971).

13) O. Tanaka and A. Takimoto, Plant \& Cell Physiol., 18, 27 (1977).

14) K. Watanabe and A. Takimoto, Plant \& Cell Physiol, 20, 847 (1979).

15) S. Kaihara, K. Watanabe and A. Takimoto, Plant \& Cell Physiol., 22, 819 (1981).

16) K. Watanabe, T. Fujita and A. Takimoto, Plant \& Cell Physiol., 22, 1469 (1981).

17) S. Fujioka, I. Yamaguchi, M. Murofushi, N. Takahashi, S. Kaihara and A. Takimoto, Plant \& Cell Physiol., 24, 235 (1983).

18) S. Sanai, Shokubutsu no Kagaku Chosetsu, 12, 59 (1977).

19) H. B. Posner, Plant Physiol, 44, 562 (1969).

20) W. S. Hillman, Amer. J. Bot., 48, 413 (1961).

21) T. Imai and T. Suzuki, Protein, Nucleic Acid and Enzyme, 15, 162 (1970).

22) H. Taguchi, "Vitamin-gaku Jikkenho II," ed, by The Vitamin Society of Japan, Tokyo Kagaku Dojin, Tokyo, 1984, pp. 356 365 .

23) O. Tanaka and A. Takimoto, Plant \& Cell Physiol., 17, 299 (1976).

24) H. Taguchi, H. Yamada, N. Ishihara, K. Okumura and Y. Shimabayashi, Vitamins (Japan), 61, 355 (1987).

25) D. G. Lynn, K. Nakanishi, S. L. Patt, J. L. Occolowitz, S. Almeida and L. S. Evans, J. Am. Chem. Soc., 100, 7759 (1978).

26) H. Taguchi and K. Iwai, Agric. Biol. Chem., 40, 385 (1976).

27) H. Taguchi, H. Yamada, N. Ishihara, K. Okumura and Y. Shimabayashi, Vitamins (Japan), 61, 445 (1987).

28) S. Nasu, K. Yamaguchi, S. Sakakibara, H. Imai and I. Ueda, Biochim. Biophys. Acta, 677, 109 (1981). 